\title{
GEOELECTRICAL INVESTIGATION OF THE SUBSURFACE USING 2D ARRAY CONFIGURATION IN UNIBEN ENVIRONS, EDO STATE, NIGERIA
}

\author{
Solomon E. O. ${ }^{1}{ }^{*}$, Ogah V. A. ${ }^{2}$ \\ ${ }^{1}$ Department of Physics, University of Benin, Benin city, Nigeria \\ ${ }^{2}$ Department of Physics, University of Benin, Benin city, Nigeria \\ DOI: 10.31364/SCIRJ/v7.i5.2019.P0519647 \\ http://dx.doi.org/10.31364/SCIRJ/v7.i5.2019.P0519647
}

\begin{abstract}
The two dimensional (2D) electrical resistivity survey was employed to investigate the subsurface layers within the engineering field in the University of Benin, Ugbowo Campus, Benin City. In this work, Wenner-Schlumberger array was used as protocol chosen for this investigated study. Field work was carried out along six (6) spreads in total in this location with length of about $230 \mathrm{~m}$ and the depth of investigation is around $43.0 \mathrm{~m}$.
\end{abstract}

After processing the result of the field work, data files were imported from a Pasi-Earth resistivity meter which had an inbuilt booster for computer interpretation using RES2DINV software to get $2 \mathrm{D}$ imaging of electrical resistivity sections. These sections reflects the subsurface complexity due to sediment heterogeneity.

The soil site is generally characterized by its low to medium to high resistivity values. While the lower resistivity values indicates the presence of sandstone and conglomerates such as fractured limestone and unconsolidated sand and gravel, the medium-high resistivity values are an indication of sediments like sandstone, limestone and shale. Higher resistivity values are associated with layers suspected to contain a mix of igneous and metamorphic rocks which range from Granite, Basalt, Slate, Marble and Quartzite. Generally, 8 distinct geoelectrical layers have been identified representing the subsurface of the study area. Resistivity values increased with depth due to decreasing soil moisture particularly at depth $19.9-43.0 \mathrm{~m}$, which makes the site suitable for construction purposes as the weathering pattern is uniform and the basement is uniformly thick.

Keywords: Geoelectric; Investigation; Heterogeneous; Resistivity; UNIBEN; Edo State

\section{Introduction}

The survey was conducted as part of experimental studies to determine the effectiveness of using parallel sets of 2-D profiles in geoelectrical resistivity imaging, with the aim of delineating various lithological units as well as determine the suitability of the site for various engineering purposes and its ground water potential.

In this research work, Wenner-Schlumberger array was used to delineate the area for various parameters of interest. Besides better horizontal coverage, the maximum depth of penetration of this array is about $15 \%$ larger than the Wenner array. The technique is extremely useful for investigations of important sites to get information on weak zones or buried channels, under the rock interface which goes undetected in seismic refraction, which terminates at rock interface.(1)

Records show that the depths of aquifers differ from place to place because of variational geo-thermal and geo-structural occurrence. $(2 ; 3)$.
The geology of Benin reveals that the entire area is underlain by sedimentary rocks. These rocks are of ages between Paleocene to recent. The sedimentary rock contains about 90 percent of sandstone and shale intercalation. It is coarse grained locally fine grained in some areas, poorly sorted, subangular to well-rounded and bears lignite streaks and wood fragment (4).The sedimentary rock of the study area constitutes the Benin formation. This has an important groundwater reservoir. Large quantity of water is obtained in a place like Benin City.

This paper is centered on 2D geoelectric investigation undertaken in UNIBEN Environs, a typical area of the Benin formation. Probing at depths of about $43 \mathrm{~m}$ below the subsurface showed that the study area is heterogeneous in nature as it transcends from sedimentary rocks to igneous and metamorphic rocks at lower depths. In areas with limited open space for a long survey line, the conventional Wenner array has a disadvantage in that there is a large reduction in 
horizontal coverage when the electrode spacing is increased in order to achieve a deeper depth of investigation. For the Wenner array each deeper data level has 3 data points less than the previous data level, while for the Wenner-Schlumberger array there is a loss of 2 data points with each deeper data level $(5 ; 6)$.

The horizontal data coverage is slightly wider than the Wenner array, but narrower than that obtained with the dipole-dipole array. The apparent resistivity for this array is given by;

$\rho_{a}=\pi n(n+1) a \frac{\Delta V}{I}$

Where a is the spacing between the $P_{1}$ and $\mathrm{P}_{2}$ electrodes and $\mathrm{n}$ is the ratio of the distances between the $c_{1}-p_{2}$ and the $P_{1}-\mathrm{P}_{2}$ electrodes. This array effectively becomes the Schlumberger array when the $\mathrm{n}$ factor is greater than 2 . Thus it is actually a combination of the Wenner and Schlumberger arrays adapted for use for an arrangement with a line of electrodes with a constant spacing (as normally used in 2-D electrical imaging)(7).

\section{Theory}

\section{Electrical Resistivity Theory}

For a single current electrode implanted at the surface of a homogeneous medium of resistivity $p$, current flows away radially (Figure 1.0).

(A)

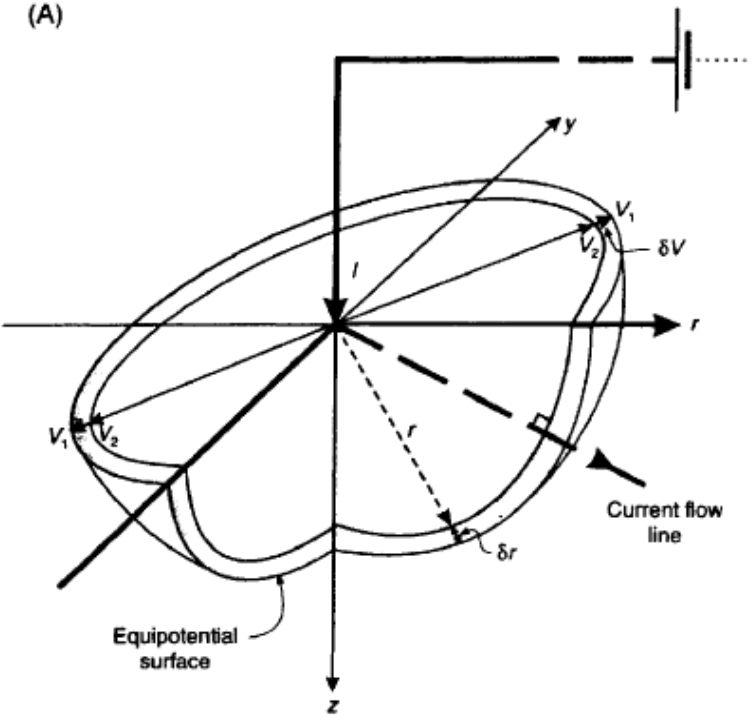

(B)

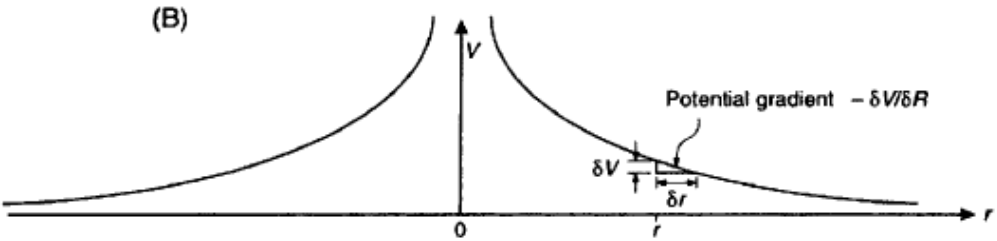

Figure 1.0 (A) Three-dimensional representation of a hemispherical equipotential shell around a point electrode on a semi-infinite, homogeneous medium. (B) Potential decay away from the point electrode
The voltage drop between any two points on the surface can be described by the potential gradient $(-\delta V / \delta x)$, which is negative because the potential decreases in the direction of current flow. Lines of equal voltage ('equipotentials') intersect the lines of equal current at right-angles. The current density $(J)$ is the current $(I)$ divided by the area over which the current is distributed (a hemisphere; $2 \pi r^{2}$ ), and so the current density decreases with increasing distance from the current source. It is possible to calculate the voltage at a distance $(r)$ from a single current point source.

The potential difference $(\delta V)$ across a hemispherical shell of incremental thickness $\delta r$ is given by:

$$
\begin{gathered}
\frac{\delta V}{\delta r}=-\rho . J \\
=-\rho \frac{I}{2 \pi r^{2}}
\end{gathered}
$$

Thus the voltage $V_{r}$ at a point $\mathrm{r}$ from the current point source is:

$$
\begin{aligned}
V_{r}=\int \delta V= & -\int \rho \frac{I}{2 \pi r^{2}} \delta r \\
= & \frac{\rho I}{2 \pi} \cdot \frac{1}{r}
\end{aligned}
$$

If, however, a current sink is added, a new potential distribution occurs (Figure 1.1)

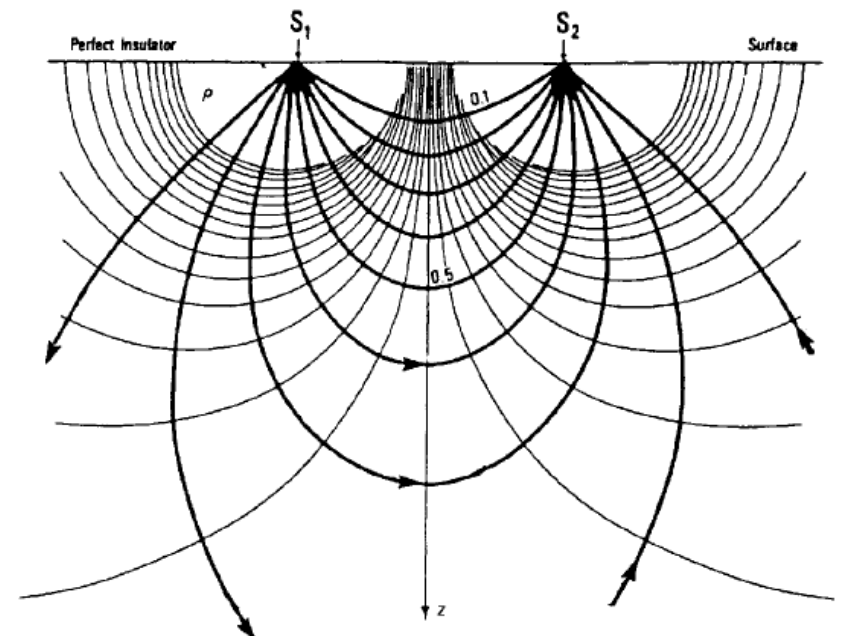

Figure 1.1 Current and equipotential lines produced by a current source and sink. (8)

For a current source and sink (Figure 2.3), the potential $V_{P}$ at any point $P$ in the ground is equal to the sum of the voltages from the two electrodes, such that:

$$
V_{p}=V_{A}+V_{B}
$$

Where $V_{A}$ and $V_{B}$ are the potential contributions from the two electrodes, $A(+1)$ and $B(-1)$. 


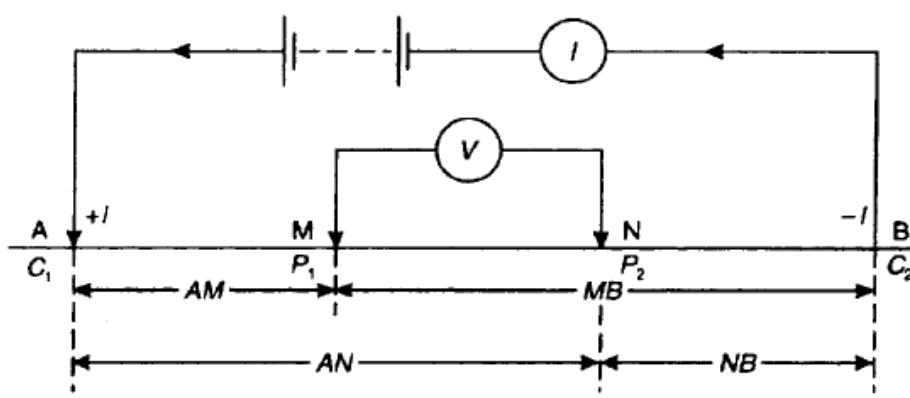

Figure 1.2 Generalised form of electrode configuration in resistivity surveys

The potentials at electrode $\mathrm{M}$ and $\mathrm{N}$ are:

$$
\begin{aligned}
V_{M}=\frac{\rho I}{2 \pi}\left[\frac{1}{A M}-\frac{1}{M B}\right], \quad V_{N} \\
=\frac{\rho I}{2 \pi}\left[\frac{1}{A N}-\frac{1}{N B}\right]
\end{aligned}
$$

However, it is far easier to measure the potential difference,

$$
\begin{aligned}
\delta V_{M N}=V_{M}-V_{N} & =\frac{\rho I}{2 \pi}\left\{\left[\frac{1}{A M}-\frac{1}{M B}\right]\right. \\
& \left.-\left[\frac{1}{A N}-\frac{1}{N B}\right]\right\}
\end{aligned}
$$

Rearranging this so that resistivity $\rho$ is the subject:

$$
\begin{aligned}
\rho=\frac{2 \pi \delta V_{M N}}{I} & \left\{\left[\frac{1}{A M}-\frac{1}{M B}\right]\right. \\
- & {\left.\left[\frac{1}{A N}-\frac{1}{N B}\right]\right\}^{-1} }
\end{aligned}
$$

\subsection{Electrode Configurations and Geometric Factors}

Equation 2.6 has two parts, namely a resistance term $(R$; units $\Omega)$ and a term that describes the geometry of the electrode configuration being used and which is known as the geometric factor ( $K$; units $\mathrm{m})$.

The geometric factor $(K)$ is defined by the expression:

$$
\begin{gathered}
K=2 \pi\left[\frac{1}{A M}-\frac{1}{M B}-\frac{1}{A N}\right. \\
\left.+\frac{1}{N B}\right]^{-1}
\end{gathered}
$$

Where the ground is not uniform, the resistivity so calculated is called the apparent resistivity $\left(\rho_{a}\right)$

$$
\rho_{a}=R K, \text { where } R=\delta V / I
$$

In reality, the sub-surface ground does not conform to a homogeneous medium and thus the resistivity obtained is no longer the 'true' resistivity but the apparent resistivity $\left(\rho_{a}\right)$ which can even be negative. It is very important to remember that the apparent resistivity is not a physical property of the sub-surface media, unlike the true resistivity. Consequently, all field resistivity data are apparent resistivity while those obtained by interpretation techniques are 'true' resistivities. $(9 ; 10)$

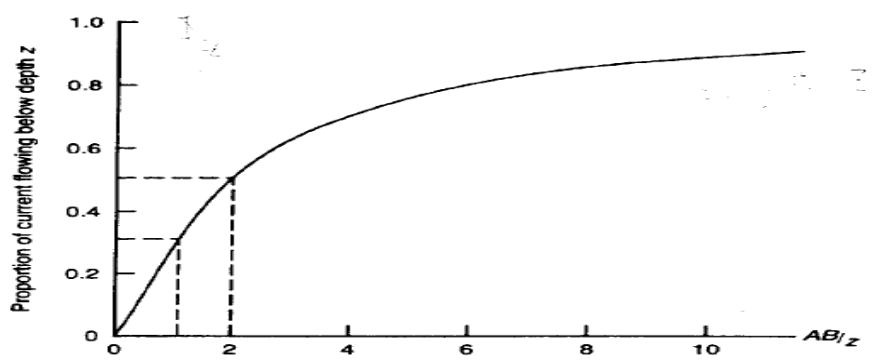

Figure1.3 Proportion of current flowing below a depth $\mathrm{z}(\mathrm{m})$; $A B$ is the current electrode half-separation

Figure 2.4 shows that, in order for at least $50 \%$ of the current to flow through an interface at a depth of $\mathrm{z}$ meters into a second medium, the current electrode separation need to be at least twice-and preferably more than three times-the depth. This has obvious practical implications, particularly when dealing with situations where the depths are of the order of several hundreds of meters, so requiring very long cable lengths that can produce undesirable inductive coupling effects. For very deep soundings where the electrode separation is more than several kilometers, telemetering the data becomes the only practical solution $(11 ; 12)$. However, it should be emphasized that it is misleading to equate the depth of penetration with the current electrode separation as a general rule of thumb in the region of a resistivity survey.

\subsection{Geometric Factor (k) for Dipole-Dipole Array}

The fundamental equation for resistivity prospecting is given by

$$
V=\frac{\rho_{a} I}{2 \pi r}
$$

$V$ is potential due to a point current source

$r$ is electrodes separation

$\rho_{a}$ is apparent resistivity

$I$ is current flow within the earth

For dipole-dipole array, potential at $P_{1}$ due to $C_{1}$ is

$$
V_{P_{1} C_{1}}=\frac{\rho_{a} I}{2 \pi n a}
$$

Potential at $P_{1}$ due to $C_{2}$ is

Total potential at $P_{1}$ is

$$
V_{P_{1} C_{2}}=\frac{\rho_{a} I}{2 \pi(a+n a)}
$$

$$
\begin{gathered}
V_{P_{1}}=\frac{\rho_{a} I}{2 \pi n a}-\frac{\rho_{a} I}{2 \pi(a+n a)}(1.9) \\
V_{P_{1}}=\frac{\rho_{a} I}{2 \pi a}\left[\frac{1}{n}-\frac{1}{1+n}\right]
\end{gathered}
$$

Potential

$$
\text { at }
$$

$$
V_{P_{2} C_{1}}=\frac{\rho_{2} I}{2 \pi(a+n a)}
$$

Potential at $P_{2}$ due to $C_{2}$ is

Total potential at $P_{2}$ is

$$
V_{P_{2} C_{2}}=\frac{\rho_{a} I}{2 \pi(2 a+n a)}
$$

$$
V_{P_{2}}=\frac{\rho_{a} I}{2 \pi(a+n a)}-\frac{\rho_{a} I}{2 \pi(2 a+n a)}(1.11)
$$

$$
\begin{gathered}
\text { Potential difference between } P_{1} \text { and } P_{2} \\
\text { is } \begin{array}{c}
\text { given } \\
V=V_{P_{1}}-V_{P_{2}}
\end{array} \\
V=\frac{\rho_{a} I}{2 \pi a}\left[\frac{1}{n}-\frac{1}{1+n}-\frac{1}{1+n}+\frac{1}{2+n}\right](1.12) \\
V=\frac{\rho_{a} I}{2 \pi a}\left[\frac{1}{n}-\frac{2}{1+n}+\frac{1}{n+2}\right](1.13)
\end{gathered}
$$




$$
\begin{gathered}
V=\frac{\rho_{a} I}{2 \pi a}\left[\frac{(1+n)(n+2)-2 n(n+2)+n(1+n)}{n(1+n)(n+2)}\right] \\
V=\frac{\rho_{a} I}{2 \pi a}\left[\frac{n+n^{2}+2+2 n-2 n^{2}-4 n+n+n^{2}}{n(1+n)(n+2)}\right] \\
V=\frac{\rho_{a} I}{2 \pi a}\left[\frac{4 n-4 n-2 n^{2}+2 n^{2}+2}{n(1+n)(n+2)}\right](1.16) \\
V=\frac{\rho_{a} I}{2 \pi a}\left[\frac{2}{n(1+n)(n+2)}\right](1.17) \\
V=\frac{\rho_{a} I}{\pi a n(1+n)(n+2)} \\
\therefore \frac{V}{I}=\frac{\rho_{a}}{\pi \operatorname{aan}(1+n)(n+2)}(1.18) \\
\operatorname{But} V=I R \\
R=\frac{V}{I}=\frac{\rho_{a}}{\pi a n(1+n)(n+2)}(1.19) \\
\rho_{a}=\pi \operatorname{TanR}(1+n)(n+2)(1.20)
\end{gathered}
$$

Let $K=\operatorname{\pi an}(1+n)(n+2)(1.21)$

$\rho_{a}=K R$

Where $K$ is the geometrical factor $(13 ; 14 ; 15)$.

PLATE I

Inverse model resistivity section for traverse 1 using Wenner-Schlumberger array

RMS Error (\%): 10.1

\section{OBSERVATION}

\section{PLATE I}

From the analysis of the result for the imaging sections of the generated profile pictures, it can be deduced that the first three layers are characterized by low resistivity values in the range $234 \Omega \mathrm{m}$ to $924 \Omega \mathrm{m}$ up to a depth of $19.9 \mathrm{~m}$ below the earth surface. These are the weathered layers and are suspected to contain sandstone and conglomerates such as fractured limestone and unconsolidated sand and gravel which are good aquifers. The next lower subsurface layers have medium resistivity values ranging from $1835 \Omega \mathrm{m}$ to $3644 \Omega \mathrm{m}$. These layers are suspected to be sedimentary rocks ranging from Sandstone, Limestone and Shale. The lower subsurface layers are heterogeneous in nature and are characterized by higher resistivity values ranging from $7236 \Omega \mathrm{m}$ to $28532 \Omega \mathrm{m}$. These layers contain a mix of igneous and metamorphic rocks which range from Granite, Basalt, Slate, Marble and Quartzite. In general, eight (8) distinct layers have been identified with increasing resistivity values spanning a total depth of $43.0 \mathrm{~m}$ below the earth surface.

\section{PLATE II}

The profile image suggests the presence of aquifers such as sandstone and conglomerates due to the low resistivity signature for some rocks at the topsoil with resistivity values

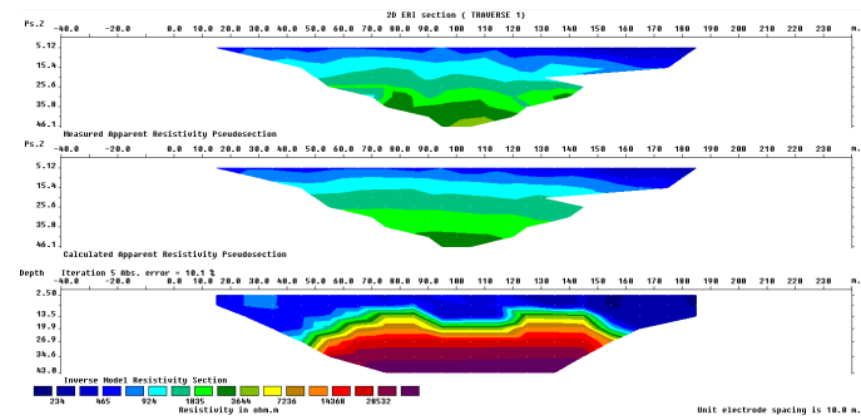

\section{PLATE II}

: Inverse model resistivity section for traverse 2 using wenner-schlumberger array

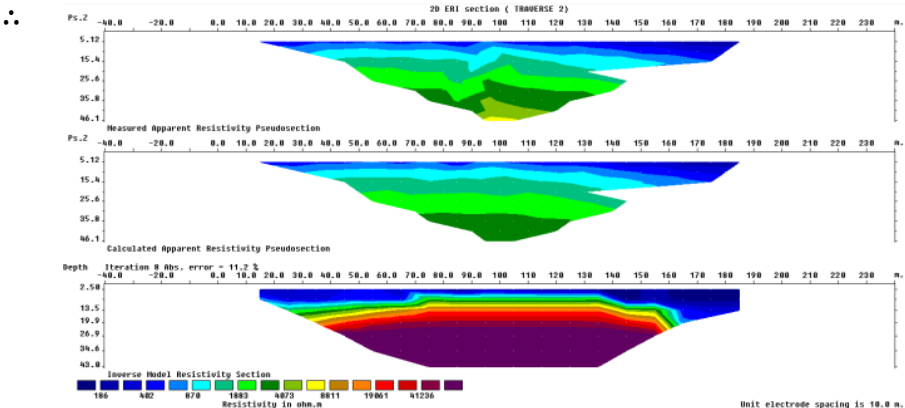

RMS (\%): 11.2

45 ranging from $186 \Omega \mathrm{m}$ to $870 \Omega \mathrm{m}$ spanning a depth of $13.5 \mathrm{~m}$ below the earth surface. The next lower subsurface layers have medium resistivity values ranging from $1883 \Omega \mathrm{m}$ to $4073 \Omega \mathrm{m}$. These layers are suspected to be sedimentary rocks ranging from Sandstone, Limestone and Shale. The lower subsurface layers are heterogeneous in nature and are characterized by higher resistivity values ranging from $8811 \Omega \mathrm{m}$ to $41236 \Omega \mathrm{m}$. These layers contain a mix of igneous and metamorphic rocks which range from Granite, Basalt, Slate, Marble and Quartzite. In general, eight (8) distinct layers have been identified with increasing resistivity values spanning a total depth of $43.0 \mathrm{~m}$ below the earth surface with a total horizontal coverage of $230 \mathrm{~m}$.

\section{CONCLUSION}

By implementing 2D resistivity imaging using WennerSchlumberger array, it is found that the Wenner-Schlumberger array gives a significant median depth of investigation and good presentation for the subsurface both in horizontal and vertical coverage with more details. Also, the weathering pattern for the location- UNIBEN Engineering field is uniform and the basement is uniformly thick. Hence, it can withstand heavy structures.

The study concludes that the analysis of the relationships between soil geotechnical properties with electrical resistivity really provides advantages for the geotechnical engineers to solve site investigation problems and any problems related to geology efficiently and economically. 


\section{REFERENCES}

[1] ZOHDY A.A. (1974): Automatic interpretation of schlumberger sounding curves. Geological survey Bulletin1313- EUS Government Printing office Washington, pp.71

[2] ZOHDY A.A, EATON C.P.\& MABEY D.R, (1974): Application of Surface geophysics to groundwater investigation - Tech.Water resources investigation, Washington, U.S Geo/Surveys

[3] CHILTON P.J. AND FOSTER S.S.D, (1995): Hydrogeological and characterization and water supply potentials of basement aquifers in tropical Africa.Hydrogeological Journal,3:36-49

[4] VAN OVERNEEREN R.A.(1989):Aquifer boundaries explored by geo-electrical measurements in the coastal plains of Yemen: A case of equivalence. Geophysics 54: $38-48$

[5] MAILET, R. (1947): The fundamental equations of electrical prospecting. Geophysics, vol.12, No.4, Pp. 529-556

[6] KELLER, G.V \& FRISCHKNECHT, (1966): Electrical methods in geophysical prospecting. Pergamon, oxford. Pp 517

[7] GRANT, F.S \& WEST, O.F (1965): Interpretation theory in New York, Pp 583
[8] Reynolds, J.M. (1998): An Introduction to Applied and Environmental Geophysics. John Wiley and Sons ltd., London UK. Second Edition. 423

[9]BHATTACHARYA, P.K. \& PATRA, H.P.(1968): Direct Current geoelectric sounding. Elsevier, Amsterdam. Pp 135

[10]FEYNMAN, R.P., LEIGHTON, R.H. \& SANDS, M. (1965): The Feynman lectures on Physics. Addison Westley reading (3 volumes)

[11] ASOKHIA M.B. (1995): Engineering Geology Services Ltd Lagos, Nigeria

[12] OTTO KOEFOED (1979): Geosounding Principles 1, ESPC-Amsterdam, Oxford, New York

[13] MILSON JOHN (1939): Field Geophysics - a handbook of geophysical society of London. John Wiley \& Sons NY, Toronto, Singapore

[14] REINHARD K. FROHLICH (1974): Combined geoelectrical and Drill-hole investigation for detecting freshwater aquifers in Northern western Missouri. Geophysics Vol. 39, pg. 340-352

[15]DORBRIN M.D and KING R.F (1976): Introduction to Geophysical prospecting. Mc Graw-Hill. Book, New York pp. 630 